\title{
Small-Molecule Photovoltaics Based on Functionalized Squaraine Donor Blends
}

\author{
Xin Xiao, Guodan Wei, Siyi Wang, Jeramy D. Zimmerman, Christopher K. Renshaw, \\ Mark E. Thompson, and Stephen R. Forrest*
}

Organic photovoltaic (OPV) cells have attracted research interest due to their potential as lightweight, low-cost, flexible sources of renewable energy. ${ }^{[1]}$ One challenge for OPVs is to find donors that lead to improved efficiencies by extending the cell photoresponse into the infrared. ${ }^{[2,3]}$ In this context, squaraines are a promising class of donors due to their large absorption coefficients in both the visible and near-infrared spectral regions, along with their large open circuit voltages $\left(V_{\text {oc }}\right)(\sim 1 \mathrm{~V})$ when paired with the acceptor, $\mathrm{C}_{60} \cdot{ }^{[4-8]}$ We have recently demonstrated efficient OPVs based on 2, 4-bis[4-( $N$-phenyl-1-naphthylamino)2,6-dihydroxyphenyl] squaraine (1-NPSQ). ${ }^{[8,9]}$ However, since the absorption peaks of $\mathrm{C}_{60}$ and 1-NPSQ lie at wavelengths of $\lambda=$ 450 and $710 \mathrm{~nm}$, respectively, and the absorption spectrum of the squaraine molecule is relatively narrow $(-100 \mathrm{~nm}$ full-widthhalf-max) compared to many other small molecule donors, there is a dip in the external quantum efficiency (EQE) spectrum between the $\mathrm{C}_{60}$ and 1-NPSQ peaks, ultimately limiting the OPV short circuit current density $\left(J_{\mathrm{sc}}\right)$. Here, we show that blending $1-N P S Q$ and the blue-shifted asymmetric donor, $\{2-[4-(N, N-$ diphenylamino)-2,6-dihydroxyphenyl]-4-diphenylamino\} squaraine (DPASQ), can partially fill the gap in EQE between $\lambda=500$ and $600 \mathrm{~nm}$, resulting in an increase in quantum efficiency in this spectral region that leads to improved device performance compared with single squaraine OPVs. Indeed, we show that solvent annealed blended cells can have power conversion efficiencies of $5.9 \pm 0.3 \%$, representing a significant improvement over single donor 1-NPSQ and DPASQ reference devices.

X. Xiao, Dr. J. D. Zimmerman, Prof. S. R. Forrest Department of Electrical Engineering

and Computer Science

University of Michigan

Ann Arbor, MI 48109, USA

E-mail: stevefor@umich.edu

G. Wei, Prof. S. R. Forrest

Department of Materials Science and Engineering

University of Michigan

Ann Arbor, MI 48109, USA

S. Wang, Prof. M. E. Thompson

Department of Chemistry

University of Southern California

Los Angeles, CA 90089, USA

C. K. Renshaw, Prof. S. R. Forrest

Departments of Physics

University of Michigan

Ann Arbor, MI 48109, USA

DOI: 10.1002/adma.201104261
Functionalized squaraines provide a versatile class of donors with exceptionally high absorption coefficients that can be tuned from the green to the infrared by simple chemical and structural modifications. The squaraine donor layers in this work consist of various mixtures of 1-NPSQ, with an absorption peak of $2.3 \times 10^{5} \mathrm{~cm}^{-1}$ at $\lambda=710 \mathrm{~nm}$, and DPASQ, with an absorption peak of $2.6 \times 10^{5} \mathrm{~cm}^{-1}$ at $\lambda=530 \mathrm{~nm}$ (see Figure 1 for the absorption spectra of 1-NPSQ and DPASQ and their corresponding molecular structural formulae) that fits within the absorption gap between $\mathrm{C}_{60}$ and 1-NPSQ. With this combination of materials, in principle, the OPV cell response can overlap the solar spectrum from 400 to $800 \mathrm{~nm}$, as required for high efficiency solar energy conversion. ${ }^{[10]}$

The $J-V$ characteristics under 1 sun AM1.5G simulated illumination of blended OPVs at various weight ratios of 1-NPSQ to DPASQ are presented in Figure 2 and Table 1 . Here, the neat 1-NPSQ (120 A thick from a $5 \mathrm{mg} \mathrm{mL}^{-1} 1$-NPSQ solution) and DPASQ (85 $\AA$ thick from a $1 \mathrm{mg} \mathrm{mL}^{-1}$ DPASQ solution) cells are used as controls. The $V_{\text {oc }}$ of neat 1-NPSQ and DPASQ cells are $0.92 \mathrm{~V}$ and $1.00 \mathrm{~V}$, respectively, whereas $V_{\text {oc }}$ of the blended cells increases with DPASQ concentration. As shown in Table 1, $V_{\text {oc }}$ increases to $1.00 \mathrm{~V}$ at a 1:2 1-NPSQ:DPASQ, which is the same as for that of the neat DPASQ cells.

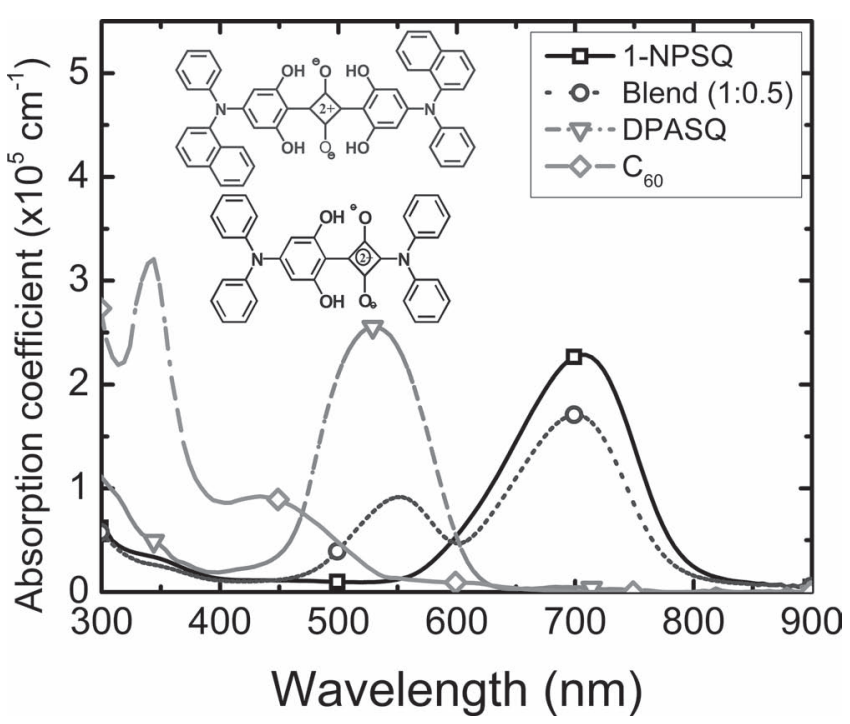

Figure 1. Absorption spectra of $\mathrm{C}_{60}, 1-\mathrm{NPSQ}$, DPASQ, and blended 1-NPSQ:DPASQ (at weight ratio of 1:0.5) films. Inset: (upper) molecular structural formula of 1-NPSQ; and (bottom) DPASQ. 


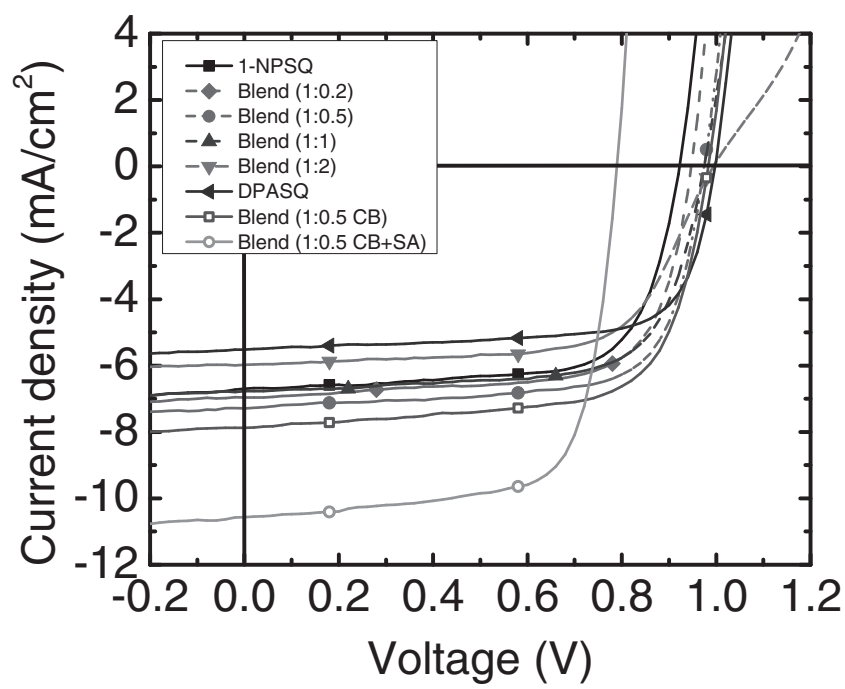

Figure 2. Current density-vs.-voltage characteristics under 1 sun, AM1.5G simulated solar illumination (spectrally corrected) for thermallyannealed, neat 1-NPSQ, DPASQ and blended donor cells at various weight ratios of 1-NPSQ to DPASQ. Also shown are characteristics for solvent-annealed $(S A)$, blended cells at a 1:0.5 ratio (here, $C B=$ compound buffer is used).

To understand the increase in $V_{\mathrm{oc}}$ of the blended cells, we used ultraviolet photoemission spectroscopy (UPS) to measure the highest occupied molecular orbital (HOMO) energies of $10 \AA$ A-thick films of 1-NPSQ and DPASQ. Films were spun cast onto ITO/glass substrates and then transferred to an ultrahigh vacuum chamber from an inert $\mathrm{N}_{2}$ environment. The measurements were performed at a base pressure $<10^{-8}$ torr using 21.22 eV He-I emission in a Thermo VG scientific Clam 4MCD analyzer system. There is $\sim 0.1 \mathrm{eV}$ shift of the low energy cutoff between 1-NPSQ and DPASQ films (see Figure 3a), while the high energy cutoffs of 1-NPSQ and DPASQ are almost identical (see Figure 3b). This suggests that the HOMO energy of DPASQ is $0.1 \mathrm{eV}$ deeper than that of 1-NPSQ; i.e., the HOMO levels of 1-NPSQ and DPASQ are at $5.3 \pm 0.1 \mathrm{eV}$ and $5.4 \pm 0.1 \mathrm{eV}$ below the vacuum level, respectively. Since $V_{\text {oc }}$ is related to the energy difference between the HOMO energy of the donor and the lowest unoccupied MO (LUMO) of the acceptor, less the polaron binding energy (i.e., the so-called interfacial energy gap, $\left.\Delta E_{\mathrm{DA}}\right)^{[11-14]}$ the deeper HOMO level of DPASQ compared with 1 -NPSQ leads to an increase in $V_{\text {oc }}$ of the former cell. Assuming a relationship between $\Delta E_{\mathrm{DA}}$ of the blend and the relative concentrations of the two donor constituents follows a linear relationship, (i.e., $\Delta E_{\mathrm{DA}}=\rho \Delta E_{\mathrm{DA}: 1-\mathrm{NPSQ}}+(1-\rho) \Delta E_{\mathrm{DA} \text { :DPASQ}}$, where $\rho$ is the weight ratio of 1-NPSQ to DPASQ, and the energies correspond to the HOMO-LUMO offsets of the individual constituents with $\mathrm{C}_{60}$, respectively), then $V_{\text {oc }}$ of the blended cells should similarly depend on $\rho$ at the donor-acceptor interface. For example, for 1:2 1-NPSQ:DPASQ, the DPASQ concentration at the surface exceeds that of 1-NPSQ, forming a nearly continuous layer at the donor/acceptor interface. Therefore, the $1: 2$ blend cells have $V_{\text {oc }}=1.00 \pm 0.02 \mathrm{~V}$, the same as that of neat DPASQ cells.

The $J_{\mathrm{sc}}$ of the blended cells compared with the neat 1-NPSQ cell increases as 1-NPSQ:DPASQ increases to 1:0.5, and then deceases as the ratio is further increased. As expected, this trend is related to changes in the EQE spectrum. As shown in Figure 4, the presence of DPASQ in the blend cells partially fills the gap between $\lambda=500$ and $600 \mathrm{~nm}$, although the quantum efficiency is significantly lower than its peak for the neat DPASQ cells. Since the absorption spectrum of DPASQ also overlaps with that of $\mathrm{C}_{60}$ (see Figure 1), the EQE between $\lambda=400$ and $500 \mathrm{~nm}$ also increases. However, the 1-NPSQ peak at $\lambda=700 \mathrm{~nm}$ deceases with $\rho$ since the number of 1-NPSQ molecules within an exciton diffusion length of the donor/ acceptor junction is reduced with increasing DPASQ concentration. When the DPASQ concentration is too high, the blended film is thicker than the excition diffusion length, leading to the decrease of the peak in EQE at $400 \mathrm{~nm}<\lambda<500 \mathrm{~nm}$. Therefore, $J_{\text {sc }}$ reaches a maximum for 1:0.5 blends, which is $\sim 8 \%$ higher when compared with neat 1-NPSQ cells.

Morphology was studied using an atomic force microscope (AFM), with surface morphologies for several films shown in Figure 5. While the neat 1-NPSQ film has a root-meansquare (rms) roughness of $\mathrm{rms}=17 \pm 1 \AA$, the surface of the 1:0.5 blend has rms $=8 \pm 1 \AA$, only half that of neat 1 -NPSQ films. As the weight ratio of DPASQ further increases, large

Table 1. Device performance of various 1-NPSQ, DPASQ and blended cells.

\begin{tabular}{|c|c|c|c|c|c|c|c|}
\hline Device & $\begin{array}{l}V_{o c} \\
{[V]}\end{array}$ & $\begin{array}{c}J_{\mathrm{sc}} \\
{\left[\mathrm{mA} \mathrm{cm}^{-2}\right]}\end{array}$ & $\mathrm{FF}$ & $\begin{array}{c}\text { PCE } \\
{[\%] \text { at } 1 \text { sun }}\end{array}$ & $\begin{array}{c}J_{\mathrm{s}} \\
{\left[\mathrm{mA} \mathrm{cm}^{-2}\right]}\end{array}$ & $n$ & $\begin{array}{c}R_{\mathrm{s}} \\
{\left[\Omega \mathrm{cm}^{2}\right]}\end{array}$ \\
\hline 1-NPSQ (TA $\left.{ }^{\mathrm{a})}\right)$ & $0.92( \pm 0.02)$ & $6.8( \pm 0.1)$ & $0.70( \pm 0.01)$ & $4.4( \pm 0.1)$ & $(7.2 \pm 0.6) \times 10^{-9}$ & $1.72( \pm 0.04)$ & $2.2( \pm 0.2)$ \\
\hline Blend(1:0.2, TA) & $0.94( \pm 0.02)$ & $7.1( \pm 0.1)$ & $0.71( \pm 0.01)$ & $4.7( \pm 0.1)$ & $(1.8 \pm 0.2) \times 10^{-8}$ & $1.89( \pm 0.04)$ & $1.4( \pm 0.1)$ \\
\hline Blend $(1: 0.5, T A)$ & $0.98( \pm 0.02)$ & $7.3( \pm 0.2)$ & $0.71( \pm 0.01)$ & $5.1( \pm 0.2)$ & $(1.3 \pm 0.2) \times 10^{-8}$ & $1.96( \pm 0.05)$ & $2.5( \pm 0.2)$ \\
\hline Blend $(1: 1, T A)$ & $0.98( \pm 0.02)$ & $7.0( \pm 0.2)$ & $0.7( \pm 0.02)$ & $4.8( \pm 0.2)$ & $(6.0 \pm 0.5) \times 10^{-8}$ & $2.16( \pm 0.07)$ & $3.0( \pm 0.3)$ \\
\hline Blend $(1: 2, T A)$ & $1.00( \pm 0.02)$ & $6.0( \pm 0.2)$ & $0.66( \pm 0.01)$ & $3.9( \pm 0.2)$ & $(1.5 \pm 0.1) \times 10^{-8}$ & $2.23( \pm 0.07)$ & $7.1( \pm 0.5)$ \\
\hline DPASQ (TA) & $1.00( \pm 0.02)$ & $5.5( \pm 0.1)$ & $0.72( \pm 0.01)$ & $4.0( \pm 0.1)$ & $(1.0 \pm 0.1) \times 10^{-10}$ & $1.58( \pm 0.03)$ & $2.9( \pm 0.2)$ \\
\hline Blend $\left.\left(1: 0.5, C B^{b}\right)+T A\right)$ & $0.98( \pm 0.02)$ & $7.8( \pm 0.2)$ & $0.69( \pm 0.01)$ & $5.2( \pm 0.2)$ & $(1.4 \pm 0.3) \times 10^{-8}$ & $1.88( \pm 0.04)$ & $1.4( \pm 0.2)$ \\
\hline Blend $\left(1: 0.5, C B+S A^{c)}\right)$ & $0.78( \pm 0.02)$ & $10.5( \pm 0.5)$ & $0.72( \pm 0.01)$ & $5.9( \pm 0.3)$ & $(1.5 \pm 0.2) \times 10^{-8}$ & $1.58( \pm 0.02)$ & $1.0( \pm 0.1)$ \\
\hline
\end{tabular}

a)TA: thermal annealed; b) $\mathrm{CB}$ : compound buffer; ${ }^{c} \mathrm{SA}$ : solvent annealed. 
(a)

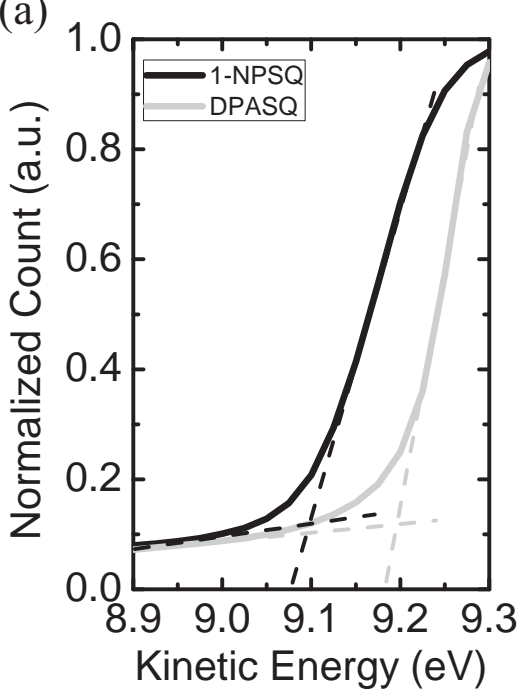

(b)

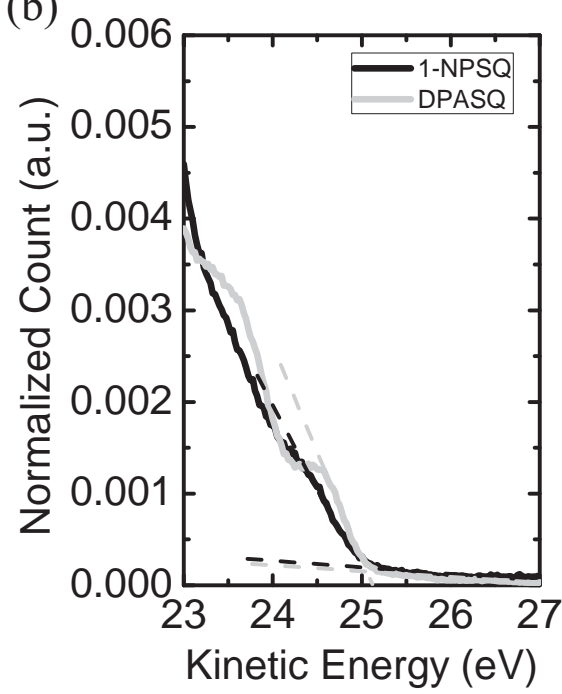

Figure 3. Ultraviolet photoelectron spectra of $10 \AA$ A-thick 1-NPSQ and DPASQ films on indiumtin-oxide-coated glass substrates. a) Low energy cutoff; and b) high energy cutoff of the films. The dashed line crossings correspond to intercepts with the energy axis.

crystallites are formed, increasing rms to $12 \pm 1 \AA$ at a $1: 2$ ratio. At high $\rho$, 1-NPSQ determines the film surface morphology, while isolated islands of DPASQ form at the higher weight ratios.

To further understand the performance of the blended cells, the modified ideal diode equation ${ }^{[12-14]}$ $J=J_{\mathrm{S}}\left[\exp \left(\frac{q\left(V_{\mathrm{a}}-J R_{\mathrm{s}}\right)}{n k_{\mathrm{b}} T}\right)-\frac{k_{\mathrm{ppd}}}{k_{\mathrm{ppd}, \mathrm{eq}}}\right]+\frac{V_{\mathrm{a}}-J R_{\mathrm{s}}}{R_{\mathrm{p}}}$ is used to fit the $J-V$ characteristics in the dark. Here, $f_{\mathrm{s}}$ is the reverse saturation current density, $q$ is the electron charge, $V_{\mathrm{a}}$ is the applied voltage, $R_{\mathrm{S}}$ is the series resistance, $n$ is the ideality factor, $k_{\mathrm{b}}$ is

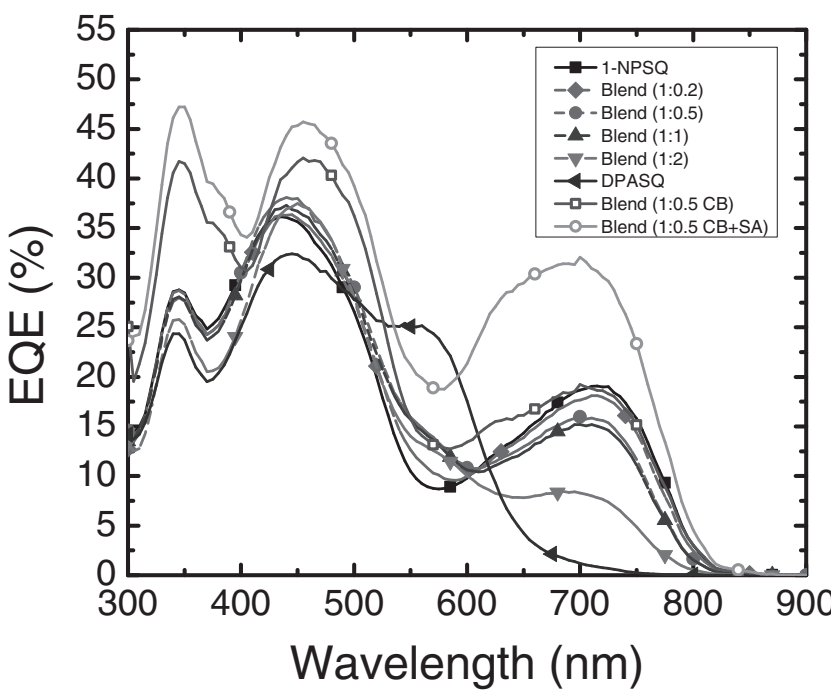

Figure 4. EQE spectra of devices in Figure 2. Note that the clearly defined feature due to exciton generation in the DPASQ film disappears for the $C B$ and $C B+S A$ films due to a significant increase in the intensity of the $\mathrm{C}_{60}$ spectra. The presence of DPASQ results in the broadening of that feature. (a)

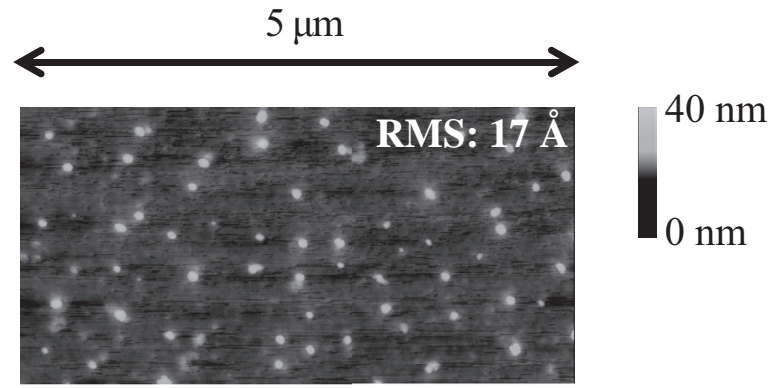

(b)

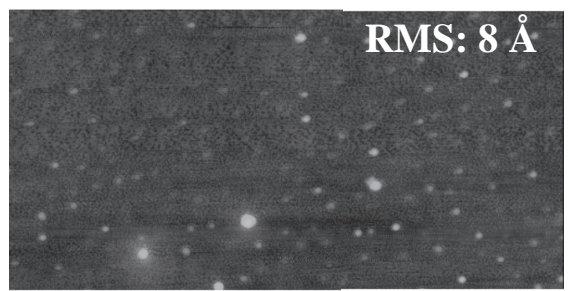

(c)

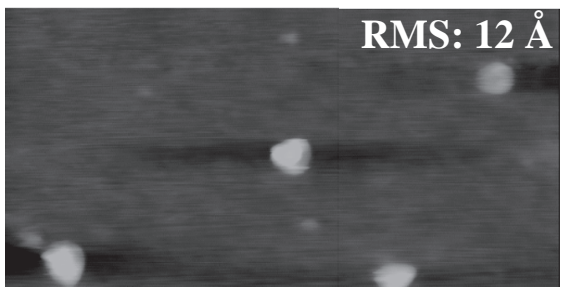

Figure 5. Atomic force microscope (AFM) images of a) a neat 1-NPSQ film; b) a 1-NPSQ:DPASQ 1:0.5 blend; and c) a 1:2 blend. Here, RMS indicates the root mean square roughness of the films in the respective images. Small-size surface clusters (possibly crystallites) were observed on neat 1-NPSQ film, which leads to RMS $=17 \AA$. The surface of 1:0.5 blend is smoother, with fewer clusters and RMS $=8 \AA$. The 1:2 blend has large clusters, with RMS $=12 \AA$. 
sun intensity for blended cells reaches $5.1 \pm 0.2 \%$ at $\rho=2$, compared with PCE $=4.4 \pm 0.1 \%$ for the neat 1 -NPSQ cells.

To further optimize cell performance, the 1:0.5 film was solvent-vapor annealed in the presence of dichloromethane vapor for $10 \mathrm{~min}$ following deposition of the $\mathrm{C}_{60}$ layer. In addition, a 1,4,5,8-napthalene-tetracarboxylic-dianhydride (NTCDA) $(150 \AA) /$ PTCBI $(50 \AA)$ compound buffer layer was used to cap the blend $/ \mathrm{C}_{60}$ cell, which further enhances the optical field distribution within the OPV active layer while blocking excitons from quenching at the cathode. ${ }^{[8]}$ For comparison, analogous thermally annealed cells with compound buffer layers were also fabricated.

The short circuit current increases from $J_{\mathrm{sc}}=7.3 \pm 0.2 \mathrm{~mA}$ $\mathrm{cm}^{-2}$ for the cells without compound buffer layers, to $J_{\mathrm{sc}}=$ $7.8 \pm 0.2 \mathrm{~mA} \mathrm{~cm}^{-2}$ for ones with such buffers, consistent with previous results. ${ }^{[8]}$ As shown in Figure 2 and Table 1, the shortcircuit current increases further, to $J_{\mathrm{sc}}=10.5 \pm 0.5 \mathrm{~mA} \mathrm{~cm}{ }^{-2}$ for solvent-annealed devices due to a significant increase of EQE in both the $\mathrm{C}_{60}$ and squaraine absorption regions. The improvement in EQE (c.f. Figure 4) upon solvent-annealing is likely due to the improvement in crystallinity of both the $\mathrm{C}_{60}$ and blended layers, which leads to an increase in exciton diffusion length and charge mobility. ${ }^{[15]}$ We note that this increase in EQE results in the disappearance of a clearly defined shoulder in the $\mathrm{C}_{60}$ peak due to exciton generation in the DPASQ. This results from the increased $C_{60}$ peak intensity, where the presence of DPASQ now results in an overall broadening of the long wavelength tail of the $\mathrm{C}_{60}$ peak. The open circuit voltage declines from $V_{\text {oc }}=0.98 \pm 0.02 \mathrm{~V}$ for thermal-annealed cells to $0.78 \pm 0.02 \mathrm{~V}$ for solvent-annealed devices, with the fill factors remaining largely unchanged. The reduction in $V_{\text {oc }}$ is attributed to the increased concentration of defects at the donor-acceptor interface during the solvent annealing process, thereby reducing $V_{\text {oc }}$ due to enhanced polaron-pair recombination. ${ }^{[16]}$ The PCE at 1 sun intensity (simulated AM $1.5 \mathrm{G}$ spectrum) increases from $\mathrm{PCE}=5.2 \pm 0.2 \%$ for thermally annealed cells to $5.9 \pm 0.3 \%$ for solvent-annealing.

The stability of the blended, unpackaged SQ OPV was tested in $\mathrm{N}_{2}$ and in ambient air under 1-sun illumination. The OPV cell in $\mathrm{N}_{2}$ retains about $70 \%$ of its initial PCE after $6 \mathrm{~h}$ while the PCE of a cell exposed to the ambient air reduces to $30 \%$ of its initial value. The degradation is primarily due to a decrease in $J_{\text {sc }}$ and $\mathrm{FF}$, while $V_{\text {oc }}$ remains approximately constant over this testing period.

In conclusion, we have demonstrated that blended 1-NPSQ:DPASQ donor layers can lead to an increased $J_{\mathrm{sc}}$ as compared to neat, single component donors. This results from increased absorption between $\lambda=450$ and $600 \mathrm{~nm}$, the location of the gap in the EQE spectrum between $\mathrm{C}_{60}$ and 1-NPSQ peaks, and enhanced $V_{\text {oc }}$ due to the deeper HOMO energy of blended film compared with neat 1-NPSQ. The power conversion efficiency at 1 sun intensity increases from $\eta_{\mathrm{p}}=4.4 \pm$ $0.1 \%$ for neat 1 -NPSQ cells, to $\eta_{\mathrm{p}}=5.1 \pm 0.2 \%$ for blend cells at an optimal 1-NPSQ:DPASQ ratio of 1:0.5. Furthermore, by solvent-annealing a blended device employing a compound, PTCBI/NTCDA exciton blocking layer, the power conversion efficiency of blended cells increases to $5.9 \pm 0.3 \%$ at 1 sun intensity due to a significant increase of $J_{\mathrm{sc}}$ in spite of a small reduction of $V_{\text {oc}}$.

\section{Experimental Section}

The OPV cells were grown on glass substrates coated with $150 \mathrm{~nm}$-thick indium tin oxide (ITO) with a sheet resistance of $20 \Omega \square^{-1}$ The substrates were cleaned in a surfactant followed by a series of organic solvents, ${ }^{[17]}$ and exposed to ultraviolet light and ozone for $10 \mathrm{~min}$ prior to loading into a high vacuum chamber with a base pressure of $1 \times 10^{-7}$ torr. Vacuum thermal evaporation (VTE) was used to deposit an $80 \AA$-thick molybdenum oxide $\left(\mathrm{MoO}_{3}\right)$ layer on the ITO surface at a rate of $0.5 \AA \mathrm{s}^{-1}$. Next, 1-NPSQ was dissolved in 1,2-dichlorobenzene (DCB) at $5 \mathrm{mg} \mathrm{mL}^{-1}$, and then added to DPASQ powder to achieve the desired weight ratios. The mixture was stirred at $100{ }^{\circ} \mathrm{C}$ in a glove box filled with ultrahigh purity $\mathrm{N}_{2}$ for $12 \mathrm{~h}$. After spin-casting the films at a rate of $3000 \mathrm{rpm}$ for $40 \mathrm{~s}$, the substrates were thermally annealed at $90{ }^{\circ} \mathrm{C}$ for 6 min under $\mathrm{N}_{2}$ atmosphere. The substrates were once more transferred to the high vacuum chamber for deposition of a $400 \AA$-thick $\mathrm{C}_{60}$ film and an $80 \AA$-thick, 3,4,9,10-perylenetetracarboxylic bis-benzimidazole (PTCBI) excition blocking and electron transporting layer, both at the rate of $1 \AA \mathrm{s}^{-1}$. A $1000 \AA$-thick $\mathrm{Ag}$ cathode layer was deposited through a shadow mask with $1 \mathrm{~mm}$-diameter circular apertures. The current density $(U)$-voltage $(V$ and EQE measurements were performed in a nitrogen glove box. The $J-V$ characteristics were measured in the dark and under simulated AM 1.5C solar illumination. The incident power intensity at one sun $\left(100 \mathrm{~mW} \mathrm{~cm}{ }^{-2}\right)$ was measured using an NRELcalibrated Si reference cell. The measured photocurrent was corrected by a spectral mismatch factor of between 0.97 and 1.0, depending on the EQE spectrum. ${ }^{[18,19]}$ Here, EQE was obtained using light from a $200 \mathrm{~Hz}$-chopped and monochromated Xe-lamp.

\section{Acknowledgements}

The authors thank Mr. Brian E. Lassiter for helpful discussions, and acknowledge the financial support from the Air Force Office of Scientific Research (SRF), the Next Generation PV Program of the United States Department of Energy (EERE) (XX GW), and Global Photonic Energy Corp. (JDZ). We also thank the Center for Solar and Thermal Energy Conversion, an Energy Frontier Research Center at the University of Michigan (Award DE-SC00000957, CKR, UPS data) and the Center for Energy Nanoscience, an Energy Frontier Research Center at the University of Southern California (Award DE-SC0001011, MET, SW, synthesis) for partial financial support.

Received: November 6, 2011

Revised: January 23, 2012

Published online: March 26, 2012

[1] S. R. Forrest, Nature 2004, 428, 911

[2] H. Y. Chen, J. H. Hou, S. Q. Zhang, Y. Y. Liang, G. W. Yang, Y. Yang, L. P. Yu, Y. Wu, G. Li, Nat. Photonics 2009, 3, 649.

[3] H. X. Shang, H. J. Fan, Y. Liu, W. P. Hu, Y. F. Li, X. W. Zhan, Adv. Mater. 2011, 23, 1554.

[4] G. D. Wei, S. Y. Wang, K. Sun, M. E. Thompson, S. R. Forrest, Adv. Energy Mater. 2011, 1, 184.

[5] G. D. Wei, R. R. Lunt, K. Sun, S. Y. Wang, M. E. Thompson, S. R. Forrest, Nano Lett. 2010, 10, 3555.

[6] S. Y. Wang, E. I. Mayo, M. D. Perez, L. Griffe, G. D. Wei, P. I. Djurovich, S. R. Forrest, M. E. Thompson, Appl. Phys. Lett. 2009, 94, 233304.

[7] G. D. Wei, S. Y. Wang, K. Renshaw, M. E. Thompson, S. R. Forrest, ACS Nano 2010, 4, 1927.

[8] B. E. Lassiter, G. D. Wei, S. Y. Wang, J. D. Zimmerman, V. V. Diev, M. E. Thompson, S. R. Forrest, Appl. Phys. Lett. 2011, 98, 243307.

[9] G. D. Wei, X. Xiao, S. Y. Wang, J. D. Zimmerman, K. Sun, V. V. Diev, M. E. Thompson, S. R. Forrest, Nano Lett. 2011, 11, 4261. 
[10] N. C. Giebink, G. P. Wiederrecht, M. R. Wasielewski, S. R. Forrest, Phys. Rev. B 2011, 83, 195326.

[11] M. D. Perez, C. Borek, S. R. Forrest, M. E. Thompson, J. Am. Chem. Soc. 2009, 131, 9281.

[12] B. P. Rand, D. P. Burk, S. R. Forrest, Phys. Rev. B 2007, 75, 115327.

[13] K. L. Mutolo, E. I. Mayo, B. P. Rand, S. R. Forrest, M. E. Thompson, J. Am. Chem. Soc. 2006, 128, 8108

[14] N. C. Giebink, G. P. Wiederrecht, M. R. Wasielewski, S. R. Forrest, Phys. Rev. B 2010, 82, 155305.
[15] R. R. Lunt, J. B. Benziger, S. R. Forrest, Adv. Mater. 2010, 22, 1233.

[16] N. C. Giebink, B. E. Lassiter, G. P. Wiederrecht, M. R. Wasielewski, S. R. Forrest, Phys. Rev. B 2010, 82, 155306.

[17] P. Peumans, S. Uchida, S. R. Forrest, Nature 2003, 425, 158.

[18] C. H. Seaman, Sol. Energy 1982, 29, 291.

[19] V. Shrotriya, G. Li, Y. Yao, T. Moriarty, K. Emery, Y. Yang, Adv. Funct. Mater. 2006, 16, 2016. 